ARTIGO ORIGINAL

\title{
Viabilidade polínica de Eucalyptus spp. com corantes específicos
}

\author{
Pollen viability of Eucalyptus spp. with specific stains
}

Ana Claudia Schllemer dos Santos ${ }^{1}$ (D), Valderês Aparecida Sousa ${ }^{2}$ (D), Simone Neumann Wendt ${ }^{1}$ (D), Ananda Virginia de Aguiar² (1)

${ }^{1}$ Universidade Tecnológica Federal do Paraná - UTFPR, Dois Vizinhos, PR, Brasil

2Embrapa Florestas, Colombo, PR, Brasil

Como citar: Santos, A. C. S., Sousa, V. A., Wendt, S. N., \& Aguiar, A. V. (2021). Viabilidade polínica de Eucalyptus spp. com corantes específicos. Scientia Forestalis, 49(131), e3588. https://doi.org/10.18671/scifor.v49n131.17

\begin{abstract}
Resumo
A hibridação tem sido intensivamente empregada nos programas de melhoramento genético desse gênero e contribuído, significativamente, para o incremento da produtividade com a incorporação de características específicas de adaptação aos materiais de interesse para o plantio. Todavia a produção de híbridos, requer a polinização controlada com pólen de qualidade, adequadamente manuseado e viável nessa ocasião. Portanto, a estimativa da viabilidade é essencial. O objetivo do presente estudo foi definir uma metodologia rápida e acurada para determinar a viabilidade do pólen de Eucalyptus usando corantes específicos. Com essa finalidade, utilizou-se o cloreto de 2,3,5, trifeniltetrazólio (TTC) nas concentrações $(0,5$ g.L.-1, 1,0 g.L.-1 e 1,5 g.L.-1) com períodos de incubação de 15, 30, 45, 60, 90 e 120 minutos, e também anilina azul em lactofenol (1 g. $\left.\mathrm{L}^{-1}\right)$ nos períodos de incubação de 5, 10, 15, 30, 45 e 60 minutos. O cloreto de 2,3,5 trifeniltetrazólio expressou a melhor viabilidade polínica, com 15 e 30 minutos, apresentando um valor muito próximo da germinação, com correlação de 0,96 . Portanto, é um corante potencial para analisar a viabilidade polínica de $E$. urophylla e E. brassiana. Para o corante azul em lactofenol, o período de imersão de 45 a 60 minutos mostrou melhores resultados. No entanto, como esse corante superestimou a viabilidade em $20 \%$, em comparação à germinação in vitro, não se recomenda o seu uso nas concentrações e períodos utilizados nesse trabalho, sendo aconselhado pesquisas com novas concentrações e período de incubação.
\end{abstract}

Palavras-chave: Germinação do pólen in vitro; Colorimetria; Tetrazólio; Anilina azul em lactofenol; Polinização controlada.

\begin{abstract}
Hybridization has been intensively used in breeding programs for Eucalyptus and has contributed, significantly, to increase the production, aside from the addition of specific adaptation characteristics of interest to plantation materials. However, the production of hybrids requires controlled pollination using pollen with an appropriate quality and viability. Therefore, accurate pollen viability estimates are essential. The aim of the present study was to define a fast and accurate methodology to determine the viability of Eucalyptus pollen using specific dyes. For this purpose, the colorimetric tests with 2,3,5 chloride, triphenyl tetrazolium (TTC) was

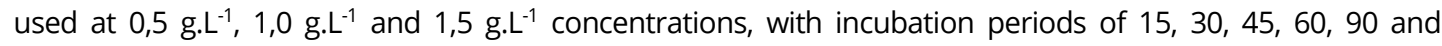
120 minutes, and also blue aniline in lactophenol (1 g.L-1) with incubation periods of 5, 10, 15, 30, 45 and 60 minutes. The 2,3,5 tripheny Itetrazolium chloride expressed the best pollen viability at 15 and 30 minutes, showing a value very close to germination, with a correlation of 0,96 . Therefore, it is a potential dye to analyze the pollen viability of $E$. urophylla and E. brassiana. For blue aniline in lactophenol, the immersion for a period of 45 to 60 minutes showed better results. However, as this dye overestimated the viability by $20 \%$, compared to in vitro germination, its use in concentrations and periods as used in the present work is not recommended. Therefore new research considering other concentrations and incubation period is recommended.
\end{abstract}

Keywords: in vitro pollen germination; Colorimetry; Tetrazolium; Blue aniline in lactophenol; Controlled pollination.

Fonte de financiamento: Empresa Brasileira de Pesquisa Agropecuária - Embrapa.

Conflito de interesse: Nada a declarar.

Autor correspondente: anaschllemer@gmail.com

Recebido: 5 agosto 2020

Aceito: 3 fevereiro 2021.

Editor: Paulo Henrique Müller Silva.

(c) (i) Este é um artigo publicado em acesso aberto (Open Access) sob a licença Creative Commons Attribution, que permite uso, distribuição e Este é um artigo publicado em acesso aberto (Open Access) sob a licença Creative Commons Attributi
reprodução em qualquer meio, sem restrições desde que o trabalho original seja corretamente citado. 


\section{INTRODUÇÃO}

As práticas silviculturais e os programas de melhoramento colaboraram para o avanço da produção florestal desse gênero, tornando-o o mais plantado e produtivo em várias regiões do país, o que proporcionou maiores ganhos genéticos ao longo dos anos, sendo superiores aos de outras culturais florestais. No melhoramento, o domínio, principalmente, das técnicas de hibridação e clonagem foram importantes para alavancar a eucaliptocultura brasileira (Assis, 2006, 2011).

A hibridação interespecífica constitui-se em uma forma rápida de obter ganhos genéticos, nos programas de melhoramento florestal, e tem sido mundialmente utilizada para o gênero Eucalyptus. Esta sofreu um enorme impulso com o advento da protoginia artificialmente induzida que permitiu o processo de polinização tornar-se eficiente e massificado, sendo hoje mundialmente utilizado (Assis et al., 2005). Por meio deste procedimento é possível reunir características produtivas e de adaptação importantes em um único indivíduo. Assim, com a complementaridade das características de crescimento, qualidade da madeira (celulose, carvão vegetal, compensados e laminados de madeira e serraria), resistência aos fatores bióticos (pragas e doenças), além da obtenção da heterose (vigor de híbrido) gera-se indivíduos com características superiores que são provenientes de diferentes procedimentos de seleção e cruzamentos (Sousa, 1988; Assis et al., 1996; Pereira et al., 2002).

Para o aproveitamento máximo do efeito da heterose, obtido no processo de hibridação, é utilizada a propagação clonal. Havendo heterose, indivíduos dominantes tendem a superar a média dos seus genitores, além de conter as características de interesse do melhor genitor utilizado (Assis et al., 1993; Fonseca et al., 2010).

Para realizar os cruzamentos controlados desejados é necessário o desenvolvimento de metodologias relacionadas a extração e beneficiamento de pólen. O manuseio do pólen inclui a coleta no estágio adequado, secagem, armazenamento e testes de viabilidade adequados. Esta última etapa é essencial para checar a real condição de germinação do pólen na obtenção de híbridos. Embora muitos testes possam ser utilizados, técnicas utilizando corantes específicos constituem-se na maneira mais rápida e prática de avaliar a viabilidade do pólen. No entanto, algumas ressalvas devem ser consideradas, como a possibilidade de superestimativa da viabilidade, podendo haver a coloração de pólens imaturos e inviáveis, em função do modo de ação de cada corante (Munhoz et al., 2008), relativamente à germinação.

Para evitar esse problema é adequado realizar comparações entre métodos para identificar quais os corantes e concentrações que estimam com maior fidelidade a viabilidade do pólen, como ocorre na germinação in vitro (Munhoz et al., 2008), de forma que haja correlação entre os métodos. Para Alvim (2008), tanto a avaliação do pólen viável pela germinação quanto pela coloração é indispensável. O uso de corantes pode tornar esse processo mais rápido, simples e barato, mas o corante utilizado não deve mascarar o valor real da germinação.

Cada corante tem ação diferenciada na atividade do grão de pólen. O cloreto de 2,3,5 trifeniltetrazólio (TTC) age na atividade das enzimas desidrogenases. Essa atividade enzimática tem relação com a sua capacidade de germinação. Quando o pólen é imerso na solução de $\Pi \mathrm{TC}$, a solução se espalha pelos tecidos, mas nas células vivas ocorre uma reação de redução, resultando na formação de um composto vermelho, conhecido como formazan, que exercerá a função do último aceptor de elétrons, substituindo o oxigênio, dentro da cadeia transportadora de elétrons na membrana mitocondrial (Derin \& Eti, 2001; França Neto et al., 1998; Hoekstra \& Bruinsma, 1975; Moore, 1973; Verleysen et al., 2004; Amutha et al., 2007).

Ao se formar, o formazan indica que há atividade respiratória e desta forma, viabilidade celular. Nesse sentido, tecidos não viáveis, ou seja, que não possuem atividade respiratória, não reagem e, portanto, não são coloridos (França Neto et al., 1998).

Assim como o TTC, o azul de anilina, em lactofenol, também é bastante utilizado para avaliar a viabilidade do pólen, agindo no citoplasma denso das células vivas, não colorindo aqueles que não possuem citoplasma ou o contém em baixa quantidade (Radford, 1986; Mulugeta et al., 1994; Domingues et al., 1999; Rigamoto \& Tyagi, 2002; Báez et al., 2002; Pline et al., 2002), o pólen viável passa então a apresentar uma coloração azul. 
Sendo assim, o objetivo do presente estudo foi definir uma metodologia rápida e acurada para determinar a viabilidade do pólen de Eucalyptus usando corantes específicos.

\section{MATERIAL E MÉTODOS}

Foi utilizado um mix de pólens de indivíduos das espécies, E. brassiana, E. urophylla. Os grãos de pólen estavam armazenados em freezer $\left(-18^{\circ} \mathrm{C}\right)$ por dois anos.

Para a reação de coloração utilizou-se o cloreto de $2,3,5$, trifeniltetrazólio (TTC) nas concentrações $\left(0,5\right.$ g.L.-1 $, 1,0$ g.L.-1 e 1,5 g.L - $\left.^{-1}\right)$ com períodos de incubação de $15,30,45$, 60, 90 e 120 minutos, e também anilina azul em lactofenol Maneval (1936) (1 g. $\mathrm{L}^{-1}$ ) nos períodos de incubação de $5,10,15,30,45$ e 60 minutos, ambos seguindo metodologia de Vianna et al. (2006).

O pólen foi distribuído sobre as lâminas, em quatro repetições, e receberam três gotas de cada corante, sendo então coberto com lamínula. Após a incubação, foram avaliados 300 grãos de pólen ao todo, por repetição, em microscópio óptico com aumento de $400 \mathrm{X}$. Esse procedimento foi repetido para ambos os corantes.

Realizou-se teste de germinação in vitro para a comparação com os testes colorimétricos. Este teste é mais realista quanto à viabilidade e capacidade do pólen emitir tubo polínico. Dessa forma, o teste in vitro foi conduzido utilizando-se a solução sugerida de Brewbaker \& Kwack (1963), com $100 \mathrm{mg} \cdot \mathrm{L}^{-1}$ de $\mathrm{H}_{3} \mathrm{BO}_{3}, 300 \mathrm{mg} \cdot \mathrm{L}^{-1}$ de $\mathrm{Ca}\left(\mathrm{NO}_{3}\right)_{2} .4 \mathrm{H}_{2} \mathrm{O}, 200 \mathrm{mg} \cdot \mathrm{L}^{-1}$ de $\mathrm{MgSO}_{4} \cdot 7 \mathrm{H}_{2} \mathrm{O}$ e $100 \mathrm{mg} \cdot \mathrm{L}^{-1}$ de $\mathrm{KNO}_{3}$, ágar e sacarose nas concentrações de 8 g. $\mathrm{L}^{-1}$ e 300 g. $\mathrm{L}^{-1}$ respectivamente. Os dados obtidos foram analisados, após a transformação em $\operatorname{arc}$ sen $\sqrt{ } \mathrm{P} / 100$, sendo $\mathrm{P}$ a porcentagem de germinação.

Para efeito de análise estatística o delineamento experimental utilizado foi de blocos completos ao acaso com quatro repetições. Para verificar a diferença entre os tratamentos (corantes e teste de germinação in vitro) os dados relativos à viabilidade e germinação foram submetidos à análise de variância (ANOVA). Para isso, aplicou-se o modelo estatístico fatorial.

As médias dos tratamentos foram comparadas pelo teste de Tukey a $5 \%$ de probabilidade, sendo analisado em esquema fatorial 3 (concentrações: 0,5 g.L.-1 $, 1,0$ g. $\left.\mathrm{L}^{-1}, 1,5 \mathrm{~g} . \mathrm{L}^{-1}\right) \times 6(15,30,45,60$, 90 e 120 minutos) para ambas as espécies com o corante TTC. Para o corante azul de anilina em lactofenol foi utilizado o fatorial 2 ( $E$. urophylla e $E$. brassiana) $\times 6(5,10,15,30,45$ e 60 minutos. Estas análises foram realizadas a partir dos procedimentos do software estatístico R@ (R Core Team, 2013). A relação entre os métodos aplicados para estimar a viabilidade do pólen também foi estimada a partir da correlação de Pearson utilizando o software livre Assistat 7.7 (Silva \& Azevedo, 2016).

\section{RESULTADOS E DISCUSSÃO}

Observaram-se diferenças significativas entre todos os tratamentos para as variáveis analisadas pelo teste $F$, havendo diferença significativa para os fatores espécie (A), corante (B) e período (C) (Tabelas 1 e 2).

Tabela 1. Resultado da ANOVA bifatorial, considerando os fatores: A) Espécie e C) Período de incubação.

\begin{tabular}{cccc}
\hline FV & GL & $\mathbf{F}$ & $\mathbf{P r}(>\mathbf{F})$ \\
\hline Bloco & 1 & 0,752 & 0,389 \\
Espécie (A) & 1 & 112,018 & $8,88 \mathrm{e}^{-16 * \star *}$ \\
Período (C) & 6 & 818,391 & $<2 \mathrm{e}^{-16 * * \star}$ \\
Espécie x período & 6 & 6,109 & $4,07 \mathrm{e}^{-05 * \star *}$ \\
Resíduo & 65 & &
\end{tabular}

***Significativo $p>0,001$ para o corante anilina azul em lactofenol $\left(1 \mathrm{~g} . \mathrm{L}^{-1}\right)$. 
Tabela 2. Resultado da ANOVA trifatorial, considerando os fatores: A) Espécie, B) Corante e C) Período de incubação.

\begin{tabular}{cccc}
\hline Fatores e interações & GL & F & Pr(>F) \\
\hline Bloco & 3 & 0,877 & 0,455657 \\
Espécie (A) & 1 & 118,853 & $<2 \mathrm{e}^{-16 * * *}$ \\
Corantes (B) & 2 & 1554,634 & $<2 \mathrm{e}^{-16 * * *}$ \\
Período (C) & 5 & 148,927 & $<2 \mathrm{e}^{-16 * * *}$ \\
Espécie x corante & 2 & 15,966 & $1,03 \mathrm{e}-06 * * *$ \\
Espécie x período & 5 & 4,219 & $0,001647 * *$ \\
Corante x período & 8 & 542,575 & $<2 \mathrm{e}^{-16 * * *}$ \\
Espécie x corante x período & 5 & 4,741 & $0,000649 * * *$ \\
Resíduo & 96 & & \\
\hline
\end{tabular}

$*_{* *}$ - Significativo $p>0,001,{ }^{*}$ - $p>0,01 e^{*}$ - $p>0,05$.

Em relação a espécie e corantes (Tabela 3), o corante que apresentou melhor resultado para ambas as espécies foi o TTC.

Tabela 3. Comparação de médias pelo teste Tukey a 5\% de probabilidade entre as duas espécies e os dois corantes avaliados.

\begin{tabular}{ccc}
\hline Espécie & Corantes & Média \\
\hline E. brassiana & TTC & $0,456 \mathrm{a}$ \\
E. brassiana & Azul & $0,457 \mathrm{a}$ \\
E. urophylla & TTC & $0,480 \mathrm{a}$ \\
E. urophylla & Azul & $0,561 \mathrm{~b}$
\end{tabular}

As três concentrações avaliadas para o corante TTC (de 0,5 a 1,5 g..-1) não diferiram estatisticamente, ao se considerar os períodos de 15 (T1) e 30 minutos (T2) (Tabela 4) (Figura 1).

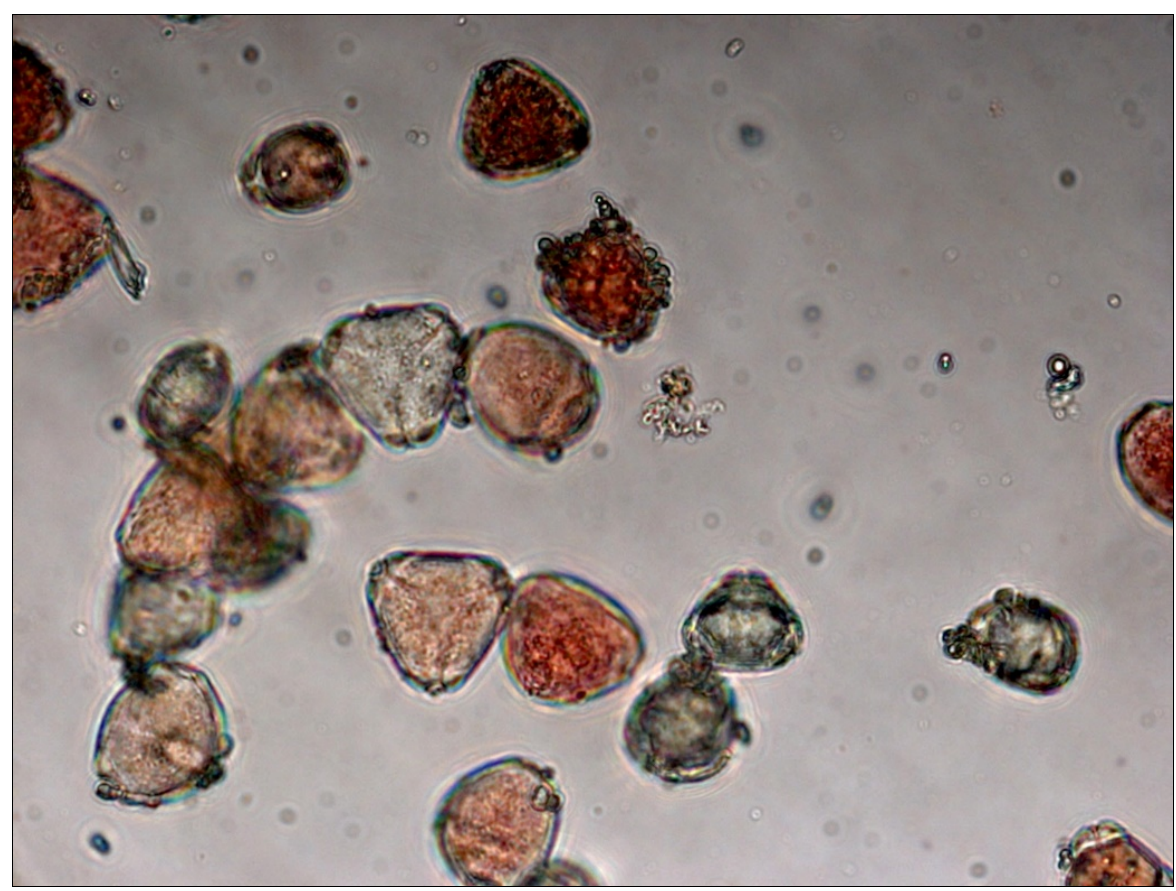

Figura 1. Teste colorimétrico com TTC para E. urophylla, os pólens viáveis são avermelhados, e pólens inviáveis são incolores. 
Tabela 4. Comparação de médias pelo teste Tukey a 5\% de probabilidade entre as duas espécies para o corante TTC, com as três concentrações testadas em $g . L^{-1} 1$ 1) 0,5, 2) 1,0, 3) 1,5 e os seis períodos de incubação avaliados em minutos.

\begin{tabular}{|c|c|c|c|}
\hline Espécie & Concentração (\%) & Período (minutos) & Média Transformada \\
\hline E. brassiana & 3 & T1 & 0,0805 a \\
\hline E. brassiana & 3 & $\mathrm{~T} 2$ & 0,0805 a \\
\hline E. brassiana & 2 & T1 & $0,0816 a$ \\
\hline E. brassiana & 2 & $\mathrm{~T} 2$ & $0,0816 \mathrm{a}$ \\
\hline E. urophylla & 3 & $\mathrm{~T} 1$ & $0,0855 a$ \\
\hline E. urophylla & 2 & $\mathrm{~T} 1$ & 0,0855 a \\
\hline E. urophylla & 3 & $\mathrm{~T} 2$ & $0,0855 a$ \\
\hline E. urophylla & 2 & $\mathrm{~T} 2$ & $0,0855 \mathrm{a}$ \\
\hline E. urophylla & 1 & $\mathrm{~T} 1$ & 0,0855 a \\
\hline E. urophylla & 1 & $\mathrm{~T} 2$ & $0,0855 \mathrm{a}$ \\
\hline E. brassiana & 1 & $\mathrm{~T} 1$ & 0,0907 a \\
\hline E. brassiana & 1 & $\mathrm{~T} 2$ & 0,0907 a \\
\hline E. brassiana & 1 & T3 & $0,3018 \mathrm{~b}$ \\
\hline E. urophylla & 1 & T3 & $0,3260 \mathrm{bc}$ \\
\hline E. brassiana & 1 & T5 & $0,4493 \mathrm{bcd}$ \\
\hline E. brassiana & 1 & T6 & $0,4503 \mathrm{bcd}$ \\
\hline E. brassiana & 1 & $\mathrm{~T} 4$ & $0,4643 \mathrm{~cd}$ \\
\hline E. brassiana & 2 & T3 & 0,4775 cde \\
\hline E. urophylla & 1 & $\mathrm{~T} 4$ & 0,5090 de \\
\hline E. urophylla & 2 & T3 & 0,5102 de \\
\hline E. urophylla & 1 & T6 & 0,5172 de \\
\hline E. urophylla & 1 & T5 & 0,5205 de \\
\hline E. brassiana & 3 & T3 & 0,5730 de \\
\hline E. urophylla & 3 & T3 & 0,6202 ef \\
\hline E. brassiana & 2 & $\mathrm{~T} 4$ & $0,7342 \mathrm{fg}$ \\
\hline E. brassiana & 2 & T6 & $0,7345 \mathrm{fg}$ \\
\hline E. brassiana & 2 & T5 & $0,7547 \mathrm{fg}$ \\
\hline E. urophylla & 2 & $\mathrm{~T} 4$ & $0,7700 \mathrm{~g}$ \\
\hline E. urophylla & 2 & T6 & $0,7800 \mathrm{~g}$ \\
\hline E. urophylla & 2 & T5 & $0,8005 \mathrm{~g}$ \\
\hline E. brassiana & 3 & T5 & $0,9717 \mathrm{~h}$ \\
\hline E. brassiana & 3 & $\mathrm{~T} 4$ & $0,9820 \mathrm{~h}$ \\
\hline E. brassiana & 3 & $\mathrm{~T} 6$ & 1,0027 hi \\
\hline E. brassiana & Testemunha & Testemunha & $0,9751 \mathrm{~h}$ \\
\hline E. urophylla & 3 & T5 & $1,1450 \mathrm{ij}$ \\
\hline E. urophylla & 3 & $\mathrm{~T} 4$ & $1,1457 \mathrm{ij}$ \\
\hline E. urophylla & 3 & $\mathrm{~T} 6$ & $1,1587 \mathrm{j}$ \\
\hline E. urophylla & Testemunha & Testemunha & $1,1494 j$ \\
\hline
\end{tabular}

Os períodos T1, T2, T3, T4, T5 e T6 correspondem a 15, 30, 45, 60, 90 e 120 minutos para o corante TTC e 5, 10, 15, 30, 45 e 60 minutos para o corante anilina azul, respectivamente.

Os melhores períodos de incubação obtidos foram 15 minutos (T1) e 30 minutos (T2) para o corante TTC em solução de 0,5 a 1,5 g. $L^{-1}$, e 45 minutos (T5) e 60 minutos (T6) para o corante anilina azul em lactofenol 1 g. $\mathrm{L}^{-1}$. Os corantes nas concentrações e períodos analisados mostraram-se 
adequados para avaliar a viabilidade polínica das espécies E. urophylla e E. brassiana, por apresentar valor muito próximo ao resultado da germinação (Figura 2 e Tabela 5).

Tabela 5. Comparação de médias pelo teste Tukey a 5\% de probabilidade entre as duas espécies, os dois corantes e os seis períodos de incubação avaliados em minutos.

\begin{tabular}{|c|c|c|c|}
\hline Espécie & Corantes & Período & Média Transformada \\
\hline E. urophylla & TTC & T1 & $0,180 \mathrm{a}$ \\
\hline E. urophylla & Azul & T6 & $0,180 \mathrm{a}$ \\
\hline E. urophylla & Azul & T5 & $0,180 \mathrm{a}$ \\
\hline E. brassiana & Azul & T6 & $0,180 \mathrm{a}$ \\
\hline E. brassiana & TTC & $\mathrm{T} 1$ & $0,180 \mathrm{a}$ \\
\hline E. brassiana & Azul & T5 & $0,180 \mathrm{a}$ \\
\hline E. urophylla & TTC & $\mathrm{T} 2$ & $0,180 \mathrm{a}$ \\
\hline E. brassiana & TTC & $\mathrm{T} 2$ & $0,180 \mathrm{a}$ \\
\hline E. brassiana & Azul & $\mathrm{T} 4$ & $0,278 a b$ \\
\hline E. urophylla & Azul & $\mathrm{T} 4$ & $0,401 \mathrm{bc}$ \\
\hline E. brassiana & Azul & T1 & $0,432 \mathrm{c}$ \\
\hline E. brassiana & TTC & T3 & $0,477 \mathrm{c}$ \\
\hline E. urophylla & TTC & T3 & $0,505 \mathrm{c}$ \\
\hline E. urophylla & Azul & $\mathrm{T} 1$ & $0,518 c$ \\
\hline E. brassiana & Azul & T3 & $0,692 \mathrm{~d}$ \\
\hline E. brassiana & TTC & $\mathrm{T} 4$ & $0,734 \mathrm{~d}$ \\
\hline E. brassiana & TTC & T6 & $0,734 d$ \\
\hline E. brassiana & TTC & T5 & $0,754 \mathrm{~d}$ \\
\hline E. urophylla & TTC & $\mathrm{T} 4$ & $0,765 \mathrm{~d}$ \\
\hline E. urophylla & TTC & T6 & $0,775 \mathrm{~d}$ \\
\hline E. urophylla & TTC & T5 & 0,795 de \\
\hline E. urophylla & Azul & T3 & 0,926 ef \\
\hline E. brassiana & Azul & $\mathrm{T} 2$ & $1,299 h$ \\
\hline E. urophylla & Azul & $\mathrm{T} 2$ & $1,484 i$ \\
\hline
\end{tabular}

Os períodos T1, T2, T3, T4, T5 e T6 correspondem a 15, 30, 45, 60, 90 e 120 minutos para o corante TTC e 5, 10, 15, 30, 45 e 60 minutos para o corante anilina azul, respectivamente.

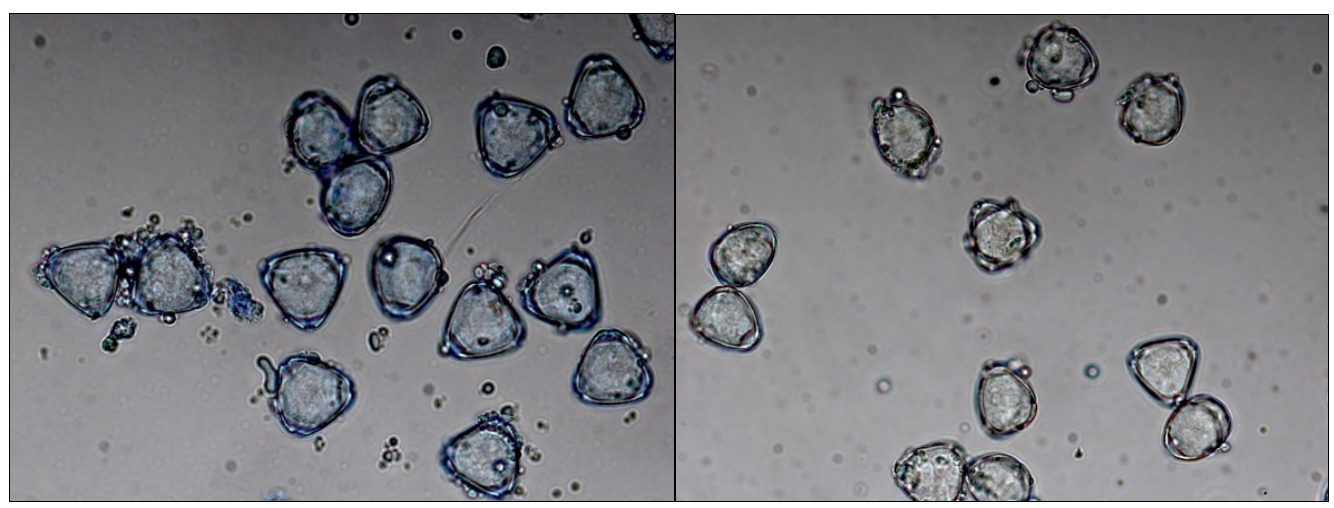

Figura 2. Teste colorimétrico com corante anilina azul em Lactofenol para $E$. urophylla. Pólens viáveis foram coloridos de azul (2A). Enquanto os inviáveis são incolores (2B).

A correlação entre o corante TTC e a germinação foi positiva e significativa. Por outro lado, a relação entre o corante azul e a germinação foi fraca (Tabela 6). Portanto, o corante 
TTC pode ser indicado para determinar a viabilidade polínica por apresentar forte correlação com a germinação do grão de pólen.

Tabela 6. Comparação entre os valores dos pares de variáveis com a correlação simples e com a parcial.

\begin{tabular}{lccc}
\hline \multicolumn{2}{c}{ PARES DE VARIÁVEIS } & \multicolumn{2}{c}{ CORRELAÇÃo } \\
\hline Azul & $\mathrm{x}$ & TTC & 0,44 \\
Azul & $\mathrm{x}$ & Germ. & 0,37 \\
TTC & $\mathrm{x}$ & Germ. & 0,96 \\
\hline
\end{tabular}

Correlação negativa e moderada entre viabilidade e germinabilidade de pólen de Elaeis guineensis foi observado por Sousa (2015). Portanto, segundo autor, o método de colorimetria, com azul de anilina de lactofenol não deve ser usado como indicativo da viabilidade polínica para esta espécie, visto que esse método pode indicar apenas a presença de conteúdo celular, o que, necessariamente, não implica a formação do tubo polínico e posterior fecundação.

Nota-se diferença entre os dois corantes analisados, comparativamente à porcentagem do teste de germinação. Para o corante TTC as porcentagens de coloração foram de $76 \%$ e $61 \%$ e de $97 \%$ e $88 \%$ para $E$. urophylla e E. brassiana, respectivamente. Já a porcentagem de germinação para ambas as espécies foi de $79 \%$ e $63 \%$, respectivamente, utilizando a solução de Brewbaker \& Kwack (1963). Este meio é considerado adequado para germinação de pólen das espécies E. urophylla e E. brassiana. Portanto, o teste de com o corante TTC propiciou uma porcentagem de coloração média $(68,5 \%)$ semelhante à encontrada no teste de germinação (71\%), mostrando-se mais realista e confiável. Já o teste com azul de anilina em lactofenol superestimou a viabilidade de germinação do pólen das espécies de Eucalyptus em 20\%, pois apresentou porcentagem de coloração superior à encontrada no teste de germinação (92,5\%), o que não é desejável pois, pode ter colorido pólen inviável (Tabela 7).

Tabela 7. Porcentagem média de germinação no teste in vitro e de coloração pelos corantes TTC anilina azul em lactofenol.

\begin{tabular}{ccccc}
\hline \multirow{2}{*}{ Espécie } & \multicolumn{3}{c}{ CORANTES } & \multirow{2}{*}{ GERMINAÇÃO } \\
\cline { 2 - 3 } & Azul & TTC & $79 \%$ \\
E. urophylla & $97 \%$ & $76 \%$ & $63 \%$ \\
E. brassiana & $88 \%$ & $61 \%$ & $71 \%$ \\
Média & $92,5 \%$ & $68,5 \%$ & \\
\hline
\end{tabular}

$O$ resultado encontrado no presente estudo foi semelhante ao encontrado por Munhoz et al. (2008) para a viabilidade polínica de Carica papaya, em que foram testados cinco corantes, cloreto de 2,3,5 trifeniltetrazólio (TTC), Alexander, carmim acético, lugol e Sudan IV. Segundo os autores, a coloração com TTC apresentou uma estimativa de viabilidade $(67,5 \%)$ equivalente ao teste de germinação in vitro, portanto, mostrou se confiável para avaliar a viabilidade polínica da espécie estudada. Os demais corantes testados por estes autores superestimaram a viabilidade do grão de pólen (> 90\%). O corante cloreto de 2,3,5 trifeniltetrazólio (TTC) também foi o mais indicado para avaliar a viabilidade polínica da mamona dentre os três corantes testados como indicadores de viabilidade do pólen dessa espécie, carmim acético 2\%, azul de tripan e TTC (Vianna et al., 2006).

Os menores valores de porcentagem de viabilidade polínica para bananeira, indicado pela coloração vermelha do pólen, também foram observados para o corante TTC por Lins et al. (2010), dentre os testes realizados com carmim acético, orceína, lugol, corante de Alexander e TTC.

Assim como no presente trabalho, o teste de viabilidade com TTC foi o mais confiável para análises da morfologia e a viabilidade polínica, em quinze genótipos de mamoneira (Ricinus communis $L$.), uma vez que os resultados obtidos com este corante foram semelhantes 
aos obtidos no teste germinação in vitro (Diamantino et al., 2016). As viabilidades polínicas de Passiflora alata Curtis (maracujá-doce) (Santos et al., 2016) e das tulipas (Damasio et al., 2016) podem ser determinadas com utilização da análise colorimétrica de TTC, pois este corante apresentou resultados eficiente, por não diferir estatisticamente da germinação in vitro.

Vários autores afirmam que o teste do TTC fornece uma estimativa confiável da viabilidade do pólen, sendo o resultado da porcentagem de coloração próximo àquele fornecido pelos testes de germinação in vitro (Huang et al., 2004), além de ser muito utilizado por ser um método relativamente rápido e simples.

Avaliando a viabilidade do pólen para a espécie Jatropha curcas (Euphorbiaceae) Abdelgadir et al. (2012), testou diferentes corantes, incluindo o TTC e azul de anilina em lactofenol. Concluíram que o TCC se mostrou mais efetivo, enquanto o corante azul de anilina em lactofenol não diferenciou o pólen viável do inviável, não sendo indicado para determinar a viabilidade do pólen da espécie estudada pelos autores.

Resultados diferentes foram obtidos por Papenfus et al. (2014), testaram a viabilidade polínica de três espécies de Amaryllidaceae, com corantes específicos e dentre eles TTC e azul de anilina em lactofenol, tendo concluído que os corantes não apresentaram diferenças significativas entre si, para as espécies estudadas.

Mayer \& Gottsberger (2000) estudando a viabilidade do pólen no gênero Silene (Caryophyllaceae), empregaram dois corantes, anilina azul em lactofenol e reação fluorocromática e observaram que o corante azul de anilina em lactofenol superestimou a viabilidade polínica, para o gênero estudado.

No estudo da viabilidade do pólen de Isatis tinctoria Asghari (2000) empregou diferentes corantes como, TTC, azul de anilina em lactofenol e acetocarmim, e verificou que o uso de azul de anilina em lactofenol, e carmim acético propiciou uma melhor estimativa da viabilidade do pólen avaliado. Portanto, os dois corantes utilizados no presente trabalho têm sido aplicados com sucesso para testar a viabilidade do pólen de diversas espécies. Porém a definição da concentração e tempo de incubação adequados devem ser, criteriosamente, investigados para cada espécie.

\section{CONCLUSÕES}

O corante cloreto de 2,3,5 trifeniltetrazólio (TTC), independente da concentração e incubado pelo período de 15 a 30 minutos foi adequado para avaliar a viabilidade do pólen de E. urophylla e E. brassiana.

O corante azul de anilina em lactofenol, por ter superestimado a viabilidade polínica de E. urophylla e E. brassiana, comparativamente à germinação in vitro, não deve ser usado nas condições do presente trabalho. Recomenda-se maiores investigações com outros tratamentos (concentrações e períodos de incubação).

\section{AGRADECIMENTOS}

Agradecemos a técnica de laboratório Marianne Bernardes da empresa Embrapa Florestas, Colombo-PR, por toda contribuição durante o desenvolvimento do trabalho, e a empresa Suzano Papel e Celulose, Itapetininga-SP por ter, gentilmente, fornecido o pólen utilizado nesse ensaio.

\section{REFERÊNCIAS BIBLIOGRÁFICAS}

Abdelgadir, H. A., Johnson, S. D., \& Van Staden, J. (2012). Pollen viability, pollen germination and pollen tube growth in the biofuel seed crop Jatropha curcas (Euphorbiaceae). South African Journal of Botany, 79, 132-139.

Alvim, P. O. (2008). Viabilidade e conservação de grãos de pólen de milho (Dissertação de mestrado). Universidade Federal de Lavras, Lavras.

Amutha, R., Muthulaksmi, S., Rani, W. B., Indira, K., \& Mareeswari, P. (2007). Physiological studies on evaluation of sunflower (Helianthus annus L.) genotypes for high temperature stress. Research Journal of Agriculture and Biological Sciences, 3(4), 245-251. 
Asghari, J. (2000). Estimation of pollen viability of metsulfuron treated dyers woad (Isatis tinctoria) for herbicide efficacy evaluation. Journal of Agricultural Science and Technology, 2, 85-93.

Assis, T. F. (2011). Hybrids and mini-cuttings: a powerful combination that has revolutionized the Eucalyptus clonal forestry. BMC Proceedings, 5(Suppl. 7).

Assis, T. F. (2006). Os propulsores da produtividade dos eucaliptos. Revista Opiniões, 14.

Assis, T. F., Abad, J. I. M., \& Aguiar, A. M. (1996). Melhoramento genético do eucalipto. Informe Agropecuário, 18, 32-51.

Assis, T. F., Bauer, J. F. S., \& Tafarel, G. (1993). Sintetização de híbridos de Eucalyptus por cruzamento controlados. Ciência Florestal, 3(1), 161-170.

Assis, T. F., Warburton, P., \& Harwood, C. (2005). Artificially induced protogyny: an advance in the controlled pollination of Eucalyptus. Australian Forestry, 68(1), 27-33.

Báez, P., Riveros, M., \& Lehnebach, C. (2002). Viability and longevity of pollen of Nothofagus species in south Chile. New Zealand Journal of Botany, 40(4), 671-678.

Brewbaker, J. L., \& Kwack, B. H. (1963). The essential role of calcium ion in pollen germination and pollen tube growth. American Journal of Botany, 50(9), 859-865.

Damasio, J. F., Santos, B. N. V., Macedo, W. A., Mello, V. S., Karsburg, I. V., Leite, D. M., \& Santos, L. C. B. (2016). Viabilidade polínica de tulipa africana. Ciência \& Tecnologia, 8(1).

Derin, K., \& Eti, S. (2001). Determination of pollen quality, quantity and effect of cross pollination on the fruit set and quality in the pomegranate. Turkish Journal of Agriculture and Forestry, 25(3), 169-173.

Diamantino, M. S. A. S., Costa, M. A. P. C., Soares, T. L., Morais, D. V., Silva, S. A., \& Souza, E. H. (2016). Morphology and viability of castor bean genotypes pollen grains. Acta Scientiarum. Agronomy, 38(1), 77-83.

Domingues, E. T., Tulmann Neto, A., \& Teófilo Sobrinho, J. (1999). Viabilidade do pólen em variedades de laranja doce. Scientia Agrícola, 56(2), 265-272.

Fonseca, S. M., Resende, M. D. V., Alfenas, A. C., Guimarães, L. M. S., Assis, T. F., \& Grattapaglia, D. (2010). Manual prático de melhoramento genético do eucalipto. Universidade Federal de Viçosa.

França Neto, J. B., Krzyzanowski, F. C., \& Costa, N. P. (1998). O teste de tetrazólio em sementes de soja. Londrina: Embrapa-CNPSo.

Hoekstra, F. A., \& Bruinsma, J. (1975). Respiration and vitality of binucleate and trinucleate pollen. Physiologia Plantarum, 34(3), 221-225.

Huang, Z., Zhu, J., Mu, X., \& Lin, J. (2004). Pollen dispersion, pollen viability and pistil receptivity in Leymus chinensis. Annals of Botany, 93(3), 295-301.

Lins, L. C. R., Soares, T. L., Costa, M. A. P., Santos-Serejo, J. A., \& Silva, S. O. (2010). Uso de diferentes corantes na determinação da viabilidade de grãos de pólen de bananeira. In $21^{\circ}$ Congresso Brasileiro de Fruticultura. Frutas: Saúde, Inovação e Responsabilidade: Anais. Natal: Sociedade Brasileira de Fruticultura

Maneval, W. E. (1936). Lacto-phenol preparations. Stain Technology, 11(1), 9-11.

Mayer, E., \& Gottsberger, G. (2000). Pollen viability in the genus Silene (Caryophyllaceae) and its evaluation by means of different test procedures. Flora, 195(4), 349-353.

Moore, R. P. (1973). Tetrazolium staining for assessing seed quality. In Seed ecology (pp. 11-23).

Mulugeta, D., Maxwell, B. D., Fay, P. K., \& Dyer, W. E. (1994). Kochia (Kochia scoparia) pollen dispersion, viability and germination. Weed Science, 42(4), 548-552.

Munhoz, M., Luz, C. F. P., Meissner Filho, P. E., Barth, O. M., \& Reinert, F. (2008). Viabilidade polínica de Carica papaya L.: uma comparação metodológica. Brazilian Journal of Botany, 31(2), 209-214.

Papenfus, H. B., Kumari, A., Kulkarni, M. G., Finnie, J. F., \& Van Staden, J. (2014). Smoke-water enhances in vitro pollen germination and tube elongation of three species of Amaryllidaceae. South African Journal of Botany, 90, 87-92.

Pereira, R. C., Davide, L. C., Ramalho, M. A. P., \& Andrade, H. B. (2002). Alternativas para aumentar a eficiência dos cruzamentos em programas de melhoramento de Eucalyptus. Cerne, 8(2), 60-69.

Pline, W. A., Edmisten, K. L., Oliver, T., Wilcut, J. W., Wells, R., \& Allen, N. S. (2002). Use of digital image analysis, viability stains, and germination assays to estimate conventional and glyphosate-resistant cotton pollen viability. Crop Science, 42(6), 2193-2200.

Radford, A. E. (1986). Fundamentals of plant systematics. Harper \& Row. 
Rigamoto, R. R., \& Tyagi, A. P. (2002). Pollen fertility status in coastal plant species of Rotuma Island. South Pacific Journal of Natural and Applied Sciences, 20(1), 30-33.

Santos, L. C. B., Fernandes, L., Macedo, W. A., Santos, B. N. V., Leite, D. M., Karsburg, I. V., \& Praça-Fonte, M. M. (2016). Uso de cloreto de trifeniltetrazólio para teste de viabilidade polínica de maracujazeiro doce. Ciência \& Tecnologia, 8(1), 1.

Silva, F. A. S., \& Azevedo, C. A. V. (2016). The Assistat Software Version 7.7 and its use in the analysis of experimental data. African Journal of Agricultural Research, 11(39), 3733-3740.

Sousa, A. S. (2015). Viabilidade e ação de lectinas na germinação in vitro de grãos de pólen de dendezeiro (Elaeis Guineensis Jacq. - Arecaceae) (Dissertação de mestrado). Universidade Estadual de Feira de Santana.

Sousa, V. A. (1988). Manejo e viabilidade do pólen de Eucalyptus spp. (Tese de doutorado). Universidade de São Paulo.

R Core Team. (2013). R: a language and environment for statistical computing.

Verleysen, H., Samyn, G., Van Bockstaele, E., \& Debergh, P. (2004). Evaluation of analytical techniques to predict viability after cryopreservation. Plant Cell, Tissue and Organ Culture, 77(1), 11-21.

Vianna, R. A. P., Bobrowskiv, L., Silva, D. S., \& Silva, S. D. (2006). Avaliação de diferentes corantes como indicadores de viabilidade do pólen de mamona. In Anais Eletrônicos do $2^{\circ}$ Congresso Brasileiro de Mamona. Aracaju.

Contribuição dos Autores: ACSS e VAS: Conceituação, curadoria de dados, análise formal, investigação, metodologia, administração do projeto, supervisão, validação, visualização, escrita - primeira redação, escrita revisão e edição; SNW: Conceituação, análise formal, investigação, metodologia, supervisão, validação, visualização, escrita - primeira redação, escrita - revisão e edição; AVA: Conceituação, curadoria de dados, análise formal, investigação, supervisão, validação, visualização, escrita - primeira redação, escrita - revisão e edição. 